\title{
Pédagogie Médicale : naissance
}
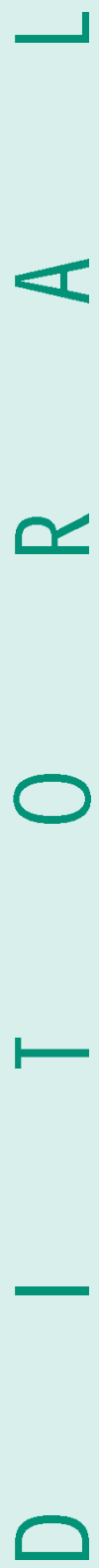

Lل」
$V$ ous avez entre les mainsle premier numéro de PÉD AGOGIE M ÉDICALE. II répond à un besoin devenu criant, celui d'une revue qui permette aux éducateurs médicaux francophones de communiquer dansleur langue, et qui soit conforme aux critères de qualité des publications internationales

La revue veut être un outil qui favorise, dans un esprit de coopération internationale, l'émergence, le renforcement \& le rayonnement d'une pédagogie francophone de qualité. Un outil qui assure la diffusion des concepts et des méthodes de formation médicale Un outil qui permet la dissémination des résultats des travaux de recherche en pédagogie.

La revue, dont la parution sera trimestrielle, est au service detous les éducateursmédicaux en formation initiale comme en formation continue Elle offre également aux responsables des facultés de médecine et des organisations de formation continue un lieu qui leur permet de partager leurs préoccupations et de proposer leurs solutions dans l'acquisition et le développement des compétences professionnelles.

PÉDAGOGIE MÉDICALE comporte plusieurs rubriques La première, intitulée "Recherches et Perspectives », contient les artides de recherche et ceux qui font le point le point dans un domaine précis. La seconde, «C oncepts et Innovations » ras semble les arti cles qui traitent de nouveaux concepts ou de nouvelles approches de formation. Elle abrite également une tribune ouverteaux auteursqui désirent exposer un point de vue original et le soumettre aux réactions des lecteurs. La troisième, "Références et Techniques » présente des textes didactiques reprenant lesgrands principesqui soustendent la miseen cuvre des actions d'éducation médicale et des textes brefs et schématiques décrivant une techniqueappliquée de présentation ou de communication. La quatrième, "N ouvellesTechnologiesÉducati ves» et destinée aux textes décrivant de nouveaux dispositifs ou outils didactiquesutilisant ou non lestechniques multimédia ou I'Internet. Enfin, la rubrique «Vie pédagogique » veut refléter le dynamismedu monde de l'éducation médicale. La revue a une composante électroniquesur un site Internet (insérer ici l'U RL du site). Ce site permettra de consulter les informations générales de la revue (adresses, directives aux auteurs, éditeur... ), les tables des matières et les résumés de différentsarticles Un forum des lecteurs, couplé au cercle des acteurs et lecteurs proposera des thèmes de discussion.

Ce numéro est le résultat d'un long processus de ges tation qui a débuté il y a plus d'une dizaine d'années avec le lancement d'une première revue, " $\mathrm{La}$ Revue d'Éducation M édicale ", par le professeur François Bonnaud, que nous remercions ic vivement pour son œuvre de pionnier. Un petit groupe d'enthousiastes a relancé le projet il y a deux à trois ans, avec le support actif de la Conférence Internationale des $D$ oyens des Facultés de $M$ édecine d'Expression Française, la CIDM EF. L'idée directrice qui a guidé cette nouvelle entreprise était de créer une revue internationale qui serve de tribune à tous leséducateurs médicaux francophones, quelles que soient leur appartenance professionnelle, leur origine géographique ou leurs modalités d'exercice.

PÉDAGOGIE M ÉDICALE a maintenant besoin de la coopération de tous. Cette coopération peut se fairetout d'abord en la faisant connaître et en incitant vos confrères ou votre institution à sabonner, et ensuite en produisant des artides, en diffusant des innovations et en partageant vos expériences et vos travaux de recherche. N ousattendons enfin de votre part des échanges avec la revue Le « cercle des lecteurs et des acteurs de la pédagogie médicale » et le forum de discussion Internet sont là pour cela. $D$ 'oreset déà nous aimeri ons connaître vosréactions concernant ce premier numéro, sa forme, son contenu, ses ambitions.

Bernard Charlin

Rédacteur en chêf

Charlinb@meddir.umontreal.ca 